\title{
Die Grenzen der Physiologie und Pathologie.
}

Von

\section{F. A. Kehrer (Heidelberg).}

Grenzbestimmungen sind stets mit Schwierigkeiten verknüpft, wenn es sich nicht um vollkommen isolierte Körper handelt. Bei zusammenhängenden, untereinander verwachsenen, ineinandergeschobenen oder eingesenkten Körpern bleibt es aber vielfach dem subjektiven Ermessen überlassen, wo man die Grenze der einzelnen ziehen soll. Ähnliches gilt, sogar noch in höherem Maasse, von unseren Begriffen, deren Abgrenzung zu zahllosen Streitigkeiten nicht bloss in der Philosophie, sondern auch in allen anderen Disziplinen geführt hat.

An dieser Stelle möchte ich versuchen, die Frage zu beantworten, $o b$ es möglich sei, zwiseben "gesund und krank", physiologiseh and pathologisch eine scharfe Grenze zu ziehen, und werde ich an einer Reihe von Beispielen zeigen, dass diese Aufgabe vielfach unlösbar ist.

Gewisse Vorgänge werden ganz allgemein in Hand- und Lehrbüchern der Pathologie und pathologischen Anatomie abgehandelt und nur nebenbei in physiologisehen Arbeiten. Dahin gebören beispielsweise Gewebsnekrose, Gewebszerreissung, Blutung, Gefässthrombose $\mathfrak{u}$. a.

Ich will nun im folgenden zeigen, dass diese und andere, allgemein als pathologisch bezeichneten Zustände und Prozesse in gewissen Lebensperioden von Pflanzen, Tieren und Menschen durchaus normal sind. Zunächst seien hier einige patbologische Zustände angeführt, welche zu den

$$
\text { angeborenen Missbildungen }
$$

gehören und darauf beruhen, dass gewisse Formen, welche in der ersten Embryonalzeit ganz normal sind, später aber durch andere ersetzt werden, noch bei Neugeborenen zutage treten. Dahin zu. rechnen sind:

Der angeborene Hydrocephalus mit Wasseransammlung in den Hirnventrikeln und die Hydromyelie, die Ansammlung von Wasser im Zentralkanal des Rückenmarkes. Es sind dies Zustände, 
welche nach dem medianen Schluss der Rückenplatten physiologisch vorkommen und in der Norm dadurch beseitigt werden, dass die Wände des Medullarrohres sowohl in der Ausdehnung der Hirnblasen wie des Rückenmarkes sich verdicken, in Nervenfasern und Ganglien sowie Neuroglia umwandeln und dadurch die eingeschlossenen Höhlen in die Spalten der Ventrikel und den engen Zentralkanal des Rückenmarkes verwandeln. Zweifellos handelt es sich bei diesen fortbestehenden Wasseransammlungen zunächst um ein Missverhältnis zwischen Sekretion der Arachnoidea und Resorption seitens der umgebenden Wände. Wir wissen aber nicht, ob dies von grösserer Durchlässigkeit der Gefässe oder von abnormer Tätigkeit der die Höhlen auskleidenden Endothelzellen abhängt.

Die verschiedenen Spaltbild ungen im Bereiche des Gesichtes, ferner die Brust-Bauchspalten und endlich die Hinterhauptund Rückgratspalten. Die Gesichtspalten beruhen darauf, dass die Stirnfortsätze und der erste Kiemenbogen in der ersten Embryonalzeit sich nicht mit ihren Rändern vereinigen. Die angeborenen Halsfisteln beruhen zum Teil auf Nichtvereinigung der Ränder der Kiemenbogen, die Brust-Bauchspalte auf dem Offenbleiben des breiten bei Beginn der Abschnürung des Visceralrohrs von der Dotterblase noch vorhandenen Brust-Bauchspaltes; der Nabelschnurbruch beruht auf Persistenz des im zweiten Monat noch vorhandenen Zustandes, wobei ein Divertikel des Bauchfells mit einer Darmschlinge vorhanden ist, welch letztere sich nicht in die Bauchhöhle zurückzieht. Bei der Hinterhaupt- und Rückgratspalte handelt es sich darum, dass die Ränder der Medullarplatten nicht zur medianen Vereinigung gelangen.

Auch die Persistenz des Foramen ovale im Septum atriorum ist auf einen physiologischen Embryonalzustand zurückzufübren.

Wenn die ursprünglichen Müller'schen Gänge ibre Duplizität beibehalten, d. h. das aus den medianen Wänden gebildete Septum nicht einschmilzt, so haben wir die verschiedenen Formen von doppeltem Uterus und Vagina.

Der Hermaphroditismus ist bei den Wirbeltieren und den Mensehen wenigstens im erwachsenen Zustand stets pathologisch, aber er ist physiologisch bei sehr vielen Pflanzen, deren Staubgefässe und Griffel in einer Blüte zusammenstehen. Bei vielen Wirbellosen, z. B. den Mollusken, haben wir ebenfalls Hermaphroditismus, wobei Ovarien und Testikel in einem Tier neben- 
einander liegen. Während aber der Hermaphroditismus bei Pflanzen zur Befruchtung und Fruchtbildung vollkommen genügt, wenn auch gewöhnlich Kreuzung eintritt, ist bei den meisten Tieren mit wenigen Ausnahmen eine gegenseitige Befruchtung $z$ weier Individuen erforderlich, um einen Embryo zu erzeugen.

In allen Fällen von pathologischem Hermaphroditismus handelt, es sich um ein fortschreitendes Wachstum in der anfünglichen Form ohne Ausgleichung der ersten Formen durch weitere Entwicklungsvorgänge.

Warum nun das Flächenwachstum der die Spalten begrenzenden Gewebe zu einer gewissen Zeit aufhört, während der übrige Embryonalkörper weiterwächst, ist unbekannt. Weiterhin seien angeführt einige Vorgänge, die allgemein als pathologisch gelten, aber ihre physiologischen Vorbilder haben. Es sind dies Veränderungen der Zirkulation und ihrer Organe sowie An-, Um-, Neu- und Rückbildungen.

\section{Zirkulationsveränderungen.}

Hy perämie.

Jede Arbeit führt bei den Tieren und Menschen zu einer Blutüberfüllung der Organe mit Erweiterung der betreffenden Kapillaren. Diese Überfüllung schwindet aber in der Regel mit Eintritt der Ruhe. Dauernde Blutüberfullung kommt nur dann vor, wenn sich die Einzelleistungen raseh folgen resp. die Reize sich dicht aneinanderschliessen und anhäufen oder die Einwirkung auf die Vasodiladatoren längere Zeit andauern.

Wir sehen zur Zeit der Geschlechtsreife sowie während der Fortpflanzung überhaupt, und zwar zunächst während der Brunst (Katamenien) nicht bloss in den Genitalien selbst, sondern auch in gewissen bei der Sexualtätigkeit beteiligten Organen eine länger dauernde Hyperämie eintreten. Was die entfernteren Organe betrifft, so möchte ich auf die Schwellung und Rötung der Kämme und Hautlappen an Kopf und Kinn unserer Hühnervögel während der Fortpflanzungszeit hinweisen. Bei den Männchen tritt diese Erscheinung am lebhaftesten hervor, weil bei ihnen die Kämme überhaupt bedeutend stärker entwickelt sind, aber auch bei den Weibchen sind zur Legezeit die Kämme und Halslappen grösser und lebhafter gerötet als sonst. 


\section{Anbildung.}

Hypertrophie (Überwachstum).

Wenn ein Gewebe oder Organ im ganzen stärker wächst als andere mit ihm verbundene oder benachbarte Teile, so kann es sich nach Virchow um Vergrösserung der Gewebszellen einfache oder unnumerische Hyperplasie der morphologischen Elemente handeln, sozusagen um Benutzung grösserer Bausteine oder Aufsetzen zahlreicherer Bausteine des gewöhnlichen Formates. Umgekehrt wird bei der Verkümmerung das Wachstum unterbrochen, oder es werden die einzelnen Elemente kleiner resp. deren Zahl vermindert: das Organ bleibt in ersterem Falle klein im Vergleich zu den weiter wachsenden übrigen Organen (gehemmtes Wachstum), oder es bildet sich in einer der beiden angegebenen Richtungen zurück.

Die Ausdrücke Hypertrophie und Atrophie stützen sich auf die Annahme, dass ein vermehrter oder verminderter Zufluss von Gewebssäften und Verbrauch der letzteren zur Organbildung die Ursache der Zu- oder Abnahme sei, daher der Ausdruck Trophie. Es ist aber noch fraglich, inwieweit das Wachstum Folge sei der gesteigerten oder verminderten Zufuhr, und nicht vielmehr auf einer ungleichen Anziehung der Gewebssäfte seitens der Zellen selbst beruhe, ob also die verschiedene Stärke des Säftestromes selber und nicht vielmehr die Attraktion der Säfte durch die wachsenden Zellen als Wachstums- odex Rückbildungsursache zu betrachten sei. Im allgemeinen kann man sagen, dass Gewebselemente und Organe dann stärker wachsen, wenn sie grössere Arbeit verrichten müssen: so hypertrophiert durch bäufige und starke Anstrengung der Muskel. Aber bei der Entwicklung ist dies Prinzip nur in beschränkter Weise gültig; denn wenn wir beispielsweise an der Keimscheibe zuerst Primitivwülste, dann Medullarwülste durch starke partielle Zellwucherungen des Ektoblasten sich erheben sehen, wenn weiterhin Augenlider, äussere Nase, Lippen, Ohrmuscheln, die Stammzellen der Extremitäten durch lokalisierte Ekto- und Mesoblastwucherungen sich bilden und an der Oberfläche erheben, so kann diese unzweifelhaft partielle Hypertrophie der einzelnen Keimhäute nicht als Folge stärkerer Arbeit betrachtet werden, weil alle diese Wucherungen erst in späterer Zeit zu leistungsfähigen Organen heranwachsen.

Wir betrachten nun die hypertrophischen Vorgänge im be- 
sonderen. Wo das normale Wachstum aufhört und die Hypertrophie, selbst Neoplasie anfängt, ist vom vergleichend-physiologischen Standpunkte nicht zu sagen, indem sowohl die Vergrösserung. wie Vermehrung der Gewebselemente an gewissen Stellen ein durchaus normaler Entwicklungsvorgang ist. Wir müssen es deshalb unentschieden lassen, ob z. B. Hervorwachsen der Kiemenbüschel am Halse der Amphibienlarven als normale Entwicklung oder als Hypertrophie der Hautgebilde oder als Neubildung zu betrachten ist. Jedenfalls kennen wir in der Entwicklungsgeschichte gewisse Vorgänge, welche füglich als Hypertrophie zu deuten sind. Dahin gehört die Entwicklung von Chorionzotten zum Fruchtkuchen. In der ersten Embryonalzeit trägt die ganze Oberfläche des Chorion zahlreiche, zerstreute Zotten und heisst es dann Chorion villosum. Später wuchern aber diese Zotten und verwandeln sich in stark verzweigte Bäumchen, welche die Placenta foetalis darstellen, und zwar an der Stelle der Eioberfläche, welche der gefässreichen Uterusschleimhaut dicht anliegt; an dem übrigen Umfange des Eies, der von der Decidua reflexa überzogen wird, atrophieren aber die Zotten, und dieser Teil wird nun zum Chorion laeve. Hand in Hand mit der Zottenatrophie geht die Rückbildung der Reflexa. Bei diesem Vorgang kann man daran denken, dass die reichlichere Säftezufuhr an der Decidua placentaris zur Zottenwucherung, die herabgesetzte Zufuhr dagegen an der Decidua reflexa zur Zottenatrophie geführt hat.

Man kann auch füglich von einer Hypertrophie des Herzens gegenüber den Gefässen reden, wenn der S-förmig gekrümmte Herzschlauch anfängt, das Blut in dem geschlossenen Gefässsystem umherzutreiben. Die anfangs indifferenten Zellen des Herzschlauches verwandeln sich dann in quergestreifte Muskelfasern, und letztere bypertrophieren in dem Maasse, als die Arbeit für die einzelnen Abteilungen des Herzens grösser wird. Auch in der ersten Lebenszeit geht diese Hypertrophie weiter, jedoch an den beiden Herzhälften sowohl in den Vorböfen wie in den Ventrikeln, in ungleicher Weise. Sie ist stärker an den Ventrikeln, als an den Vorhöfen, aber auch an ersteren wieder ungleich, indem nach der Obliteration des Ductus Botalli, die während der Fötalzeit an dem Umtrieb des Aortenblutes gleichbeteiligten Ventrikel von jetzt an ungleiche Arbeit leisten. Denn der rechte Ventrikel treibt bloss einen Teil des Blutes durch die relativ kleinen Lungen, der linke Ventrikel aber das Blut durch den grösseren Teil des Körpers. 


\section{Umbildungen (Metaplasien).}

Umwandlungen einer Zellform in eine andere mit zum Teil vollständiger Veränderung der Form und Funktion kommen bei der Embryonalentwicklung regelmässig vor. So verwandeln sich die Zellen des äusseren Keimblattes oder Ektoblasts teils in die Oberhautzellen mit ihren Drüsen und Anhängen, teils, und zwar soweit sie dem Medullahrrohr angehören, in Ganglienzellen und Nervenfasern. Die Zellen des Mesoblasts gehen uber in die endothelialen Auskleidungen der Brust und Leibeshöhle, in die Epithelien der uropoëtischen- und Genitalorgane, ferner in Knorpel, Knochen, Muskelfasern, Sehnen, Gelenkkapseln, Bindegewebszellen usw. Die Zellen des Entoblasts endlich gehen über in die Epithelien des Darmkanals nebst Leber und Pankreas, des Respirationstraktus einschliesslich der Thyreoidea, der Allantois usw. Tritt nun eine Vermehrung von Epithelzellen ein, so dass sich geschichtetes Epithel entwickelt, so gilt als allgemeine Regel, dass die tiefsten der Bindegewebsschicht aufliegenden Zellen zu stehenden Zylindern, die mittleren durch Teilung der vertikalgestellten Kernspindel sich bildenden rund oder polyedrisch werden, die oberflächlichen ältesten Zellen aber, welche entweder frei liegen oder die Lichtung eines Ganges oder Hohlraumes auskleiden, sich abplatten.

Diese physiologische Aufeinanderfolge der Zellformen in den einzelnen Schichten beobachten wir auch pathologischerweise auf Schleimbäuten, welche sonst nur Zylinderepithel tragen, z. B. auf der Gebärmutter-, Harnröhren-, Harn- und Gallenblasenschleimhaut sowie an der Oberfläche und an der Innenseite drüsenartiger Wucherungen von Carcinomen. Es ahmt also hier der pathologische Vorgang den physiologischen nach. Ähnliches gilt auch für die aus den Abkömmlingen des mittleren und unteren Keimblattes entstehenden pathologischen Bildungen, wobei vielfach einzelne Zellgruppen bestimmter Form in krankhafte, aber formell vielfach veränderte Neubildungen übergehen.

\section{Neoplasien.}

Wir betrachten hier nur diejenigen Arten physiologischer Neubildung, welche in bestimmten Lebensaltern oder nur beim männlichen oder weiblichen Geschlechte vorkommen, und zwar das Hervorsprossen äusserer Kiemen in Form einzelner Fäden oder Büschel an beiden Seiten des Halses bei jungen Amphibienlarven 
(Fröschen, Salamandern). Diese bestehen aus einem Bindegewebsgerüste mit schlingenartig umbiegenden oder netzförmig zusammenhängenden Kapillaren und einem Überzug von Epithel. Während nun bei unseren Fröschen und Tritonen diese äusseren Kiemen nur eine gewisse Zeit bis zur Ausbildung der Lungen als Respirationsorgane fungieren und dann durch Atrophie schwinden, bleiben sie beim mexikanischen Axolotl zeitlebens besteben.

Ein physiologisches Vorbild für die pathologisehen Hauthörner bei Menschen und Säugetieren ist die Entwicklung der Hörner bei den Cavicornien sowie an den Beinen der Vögel. Bei den Cavicornien wächst aus der Schädeldecke, und zwar symmetrisch, ein konischer Knochenzapfen hervor, eine förmliche von Knochenhaut überzogene Exostose. An deren ganzem Umfang wuchert die Epidermis nun beträehtlich und erzeugt das eigentliche Horn. Diese Hornbildung beginnt bei jungen Tieren und schreitet später in einer mit dem Gesehlecht wechselnden Weise voran. Während aber z. B. nur bei den Widdern schneckenartige Hörner sich bilden, fehlen dem kastrierten und weiblichen Schaf die Hörner überhaupt oder sind höchstens rudimentär. Beim Genus Bos entwickeln sich, abgesehen von der ungehörnten phylogenetisch primären Rasse, bei beiden Geschlechtern Hörner, die beim Bullen kurz bleiben, bei der Kuh länger und dünner werden und beim kastrierten Ochsen, besonders. bei der ungarischen Rasse, eine ausserordentliche Länge erreichen. Ferner ist hierher zu zäblen die Bildung eines Hauthorns auf der Nasenspitze des Nashorns. Endlich sei erwähnt, dass sich bei den reifen Früchten mancher Reptilien und Vögel ein kleiner, spitzer Oberhautzapfen auf der Nasenspitze resp. dem Oberschnabel entwickelt, der zum Durchbohren der Schalenhaut und Schale dient und bald abfällt.

Eine eigentümliche Warzenbildung durch Wucherung von Hautpapillen entwickelt sich an der Unterseite der Daumenballen der Vorderfüsse männlicher Frösche zur Laichzeit. Da die männlichen Frösche mit ihren Vorderfüssen den Oberbauch der Weibchen umfassen und diese Umarmung erst mit vollendetem Austritt der Laichschnüre und der Begattung aufhört, also längere Zeit andauert, scheinen diese böckerigen Warzen dazu bestimmt zu sein, das Abgleiten von der glatten Haut des Weibchens zu verhindern.

Ein Beispiel von Exostosenbildung bieten die geweihtragenden Wiederkäuer. Bei diesen sprossen konische Knochen- 
zapfen von behaarter Haut überzogen schon vor Beginn der Geschlechtsreife hervor als förmliche Exostosen, durchbohren später die Haut und ragen als zwei an der Basis einfache, nach oben sich verästelnde Geweihe an der Stirn hervor. Durch fortgesetztes periosteales Wachstum an ihrer von Periost überzogenen Basis werden sie successive länger und dicker.

Eine ganz eigentümliche Geschwulstbildung sehen wir in den Ovarien der Säugetiere vorkommen. Sie besteht in der Entwicklung des gelben Körpers (Corpus luteum) nach der Berstung eines Gra a f'schen Follikels. Die grossen Lutëinzellen, die beim Menschen und manchen Säugern gelbe, durch Lutëin gefärbte Fettkörnchen enthalten, wuchern mit der Membrana propria, in der sie eingeschlossen sind, reichlich und bilden schliesslich einen rundlichen, teils blass-fleischfarbenen, teils gelben Tumor: das Corpus luteum, das manchmal, doch nicht immer und nicht bei allen Säugetieren das bei der Berstung entstandene Blutgerinsel enthält. Nachdem dieser Tumor seine volle Grösse erlangt hat, bildet er sich früher oder später durch Fettmetamorphose zurück. Da die Lutëinzellen, die seine Hauptmasse ausmachen, Bindegewebszellen übrigens förmliche Mastzellen sind, so könnte man von einer menstruellen transitorischen Sarkombildung gutartigen Charakters reden. Dieses. Sarkom hat unzweifelhaft Ähnlichkeit mit dem echten Deciduom S a e n g e r's, unterscheidet sich aber wesentlich von diesem durch seine regelmässig eintretende regressive Metamorphose, während bekanntlich das maligne Deciduom weiterwuchert und meist rasch zum Tode führt.

Die Corpora lutea scheinen die Bedeutung zu haben, dass sich in der ersten Hälfte des menstrualen Intervalls massenhaft Lutëinzellen bilden, die in der zweiten Hälfte der Einschmelzung resp. Fettmetamorphose anheimfalıen. Der dabei gebildete chemische Körper (Lutein) erregt nun wahrscheinlich in der zweiten Hälfte des Intervalls die genitalen Gefässzentren derart, dass schliesslich eine allgemeine Genitalhyperämie mit Menstruation und Ovulation zustande kommt. Wenn diese Ansicht richtig ist, so haben wir in den Corpora lutea ein Beispiel vor uns von einer Neubildung, die für die Genitalien sowohl wie für den übrigen Körper von grosser Bedeutung und ein notwendiges Glied in der Kette der Fortpflanzungsvorgänge ist.

Wir könnten nun von diesem Beispiel aus weitergehend uns vorstellen, dass die Neoplasmen überhaupt für Organ und Organismus zunächst nicht von deletärer Bedeutung, sondern von hoher physiologischer Wichtigkeit seien. Da jede Organisation auf Erzeugung eines für den Teil oder das Ganze wichtigen Produkts hinausläuft, da sozusagen die Natur nichts Unnützes oder dem Organismns Zweckwidriges schafft, so könnte man auch die Neoplasmen als für gewisse Zwecke 
wichtige Gebilde betrachten, und zwar in einem doppelten Sinne: sie könnten allzu reichlich zugeführte Säfte oder Einzelbestandteile derselben zurückhalten, gleichsam aus dem Kreislauf ausschalten und dadurch für den Körper unschädlich machen. Oder es könnten sich in ihnen Stoffe bilden, die für den übrigen Körper nützlich, selbst notwendig sind, ähnlich wie in der Schilddrüse der Thymus, den Nebennieren, der Milz und anderen sogenannten Blutgefässdrüsen mit innerer $\mathrm{Ab}$ sonderung. Beweisen lässt sich in dieser Beziehung vorerst noch nichts wegen Mangelhaftigkeit unserer Kenntnisse; aber wir können vielleicht mit dem Gedanken weiter kommen, dass Neoplasmen nicht, wie man immer geglaubt, schädlich und demnach operativ zu entfernen, sondern mindestens anfänglich auch für den Körper nützlich seien.

\section{Rückbildungsvorgänge.}

\section{Dehiszenz.}

Dehiszenz ist das Aufplatzen an praeformierten Nähten. Sie kommt vielfach bei Pflanzen vor, zumal an den Samenkapseln, wo sich verdickte Nähte finden, in deren Mitte die Kapsel platzt, worauf die Samen ausgestreut werden. Auch bei den Metamorphosen der Insekten begegnen wir vielfach Nähten an den Hüllen, an denen die ausschlüpfende Made oder das aus der Puppe ausschlüpfende vollkommene Insekt seine Schale sprengt.

Endlich sei die Dehiszenz der Augenlider erwähnt, welche bei jungen Vögeln und Säugetieren bald vor, bald nach der Geburt erfolgt und die Lidspalte herstellt. Hier geht eine Verhornung der Epithelschicht, welche die Oberhaut mit dem Conjunktivalepithel verbindet und sich zwischen die Tarsalränder der Lider einschiebt, der Eröffnung voraus.

\section{Perforation.}

Als physiologisches Vorbild der Perforation, welche uns z. B. beim Durchbruch der Magen- und Darmgeschwüre in die Bauchhöhle, der Lungenkavernen in die Brusthöhle, beim Durchbruch von Abszessen nach aussen und innen vielfach entgegentritt, können wir die Durchbohrung der Eihüllen bei der Keimung der Pflanzen wie bei dem Ausschlüpfen der Tiere betrachten. Es kann hierbei die in die Eihülle eingeschlossene Made oder Larve die Hülle durchschneiden, durchnagen oder durch Andrücken des Kopfes eröffnen.

Weitere Beispiele von Perforation liefern das Durchbrechen der Zähne durch das die Zahnkrone als feine Lamelle überdeckende Zahnfleisch beim ersten Zahnen sowie endlich das Durchbrechen der Geweihe bei gewissen Widerkäuern durch die mit Haaren bedeckte und allmählich sich verdünnende Leder- und Oberhaut. 


\section{Zerreissung.}

Zerreissungen können nach folgenden Mechanismen zustande kommen :

1. Durch Überdehnung, sei es, dass ein Teil festgehalten und das Gewebe nach einer Richtung gezogen wird,

2. durch exzentrische Dehnung nach entgegengesetzten Richtungen,

3. durch konzentrisches Zusammendrücken in der Richtung der Länge oder Fläche,

4. durch einen Druck rechtwinklich zu einer Fläche, wobei der gedrückte Teil aus dem Zusammenhang mit dem benachbarten herausgerückt wird.

Die pathologische Ruptur findet ihr physiologisches Vorbild in der Sprengung der Samenhüllen beim Keimen der Pflanzen sowie in der Zerreissung der Eihaut an anatomisch nicht vorbezeichneten Stellen und beginnt bei den Insekten gewöhnlich in der Mittellinie an der Rückenhaut. Der mediane Spalt setzt sich nach vorn und hinten weiter fort, worauf dann die ausschlüpfende Larve oder Nymphe oder das vollkommene Geschöpf aus dem zurückbleibenden Balg ausschlüpft. Bei den meisten Wirbeltieren wird die den Embryo umhüllende Eihaut an dem Kopfende durch Andrücken des Kopfes oder des Schnabels spaltförmig oder unregelmässig zerrissen. Die Vogelembryonen durchbohren mit der Schnabelspitze an der Luftkammer zuerst die Schalenhaut und sprengen dann die harte Schale durch Picken von inven ab, worauf der Kopfteil der letzteren durch Anstemmen des Körpers an die Innenseite der Schale abgehoben wird. Bei den Säugetieren, einschliesslich der Menschen, wird das Sprengen der Fruchtblase entweder durch gesteigerten Inhaltsdruck des Fruchtwassers an deren vorliegendem Ende, meist mittelbar durch die Zusammenziebung des Fruchthalters besorgt, oder es zerreisst die in unverletzten Eihäuten geborene Frucht mittelst ihrer Bewegungen oder ihrer Krallen den Eisack. Reichen dazu die Mittel der Frucht nicht aus, so hilft das Muttertier durch Zerbeissen oder Belecken der Eihäute resp. der hilfeleistende Mensch mit Fingernägeln oder Schere nach, um Erstickung des Neugeborenen zu verbindern.

Noch sei hier einer anderen Erscheinung gedacht: der schon im Intrauterinleben erfolgenden Zerreissung der Pupillarmembran, welche wahrscheinlich durch Bewegungen der Irismuskeln geschieht. 
Endlich sei noch die Zerreissung des $\mathrm{Nabelstranges}$ nach Austritt der Frucht aus den mütterlichen Genitalien erwähnt. Sie erfolgt an verschiedenen Stellen des Stranges entweder bei stehender Mutter durch Herabfallen des Neugeborenen auf den Boden, wobei zu bemerken ist, dass das Gewicht des neugeborenen Kindes bei einer Fallhöhe von $0,5 \mathrm{~m}$ zur Zerreissung genügt, oder sie erfolgt beim Aufstehen der im Sitzen oder Liegen niedergekommenen Mutter. Die meisten Säugetiere zerbeissen den Strang, der Mensch durchtrennt ihn mit den Händen oder scharfen Werkzeugen.

$\mathrm{Zu}$ den physiologischen Zerreissungen gehört auch die Berstung des Gra af' schen Follikels bei der Menstruation. Infolge reichlicher Ausscheidung von Liquor folliculi werden die Follikelwände an einer Stelle, die ich als Macula pellucida bezeichnet habe, so stark gespannt, dass sie schliesslich einreissen. Es bildet sich hier eine spalt- oder sternförmige Öffnung, und der Follikel fällt zusammen.

Weitere Beispiele von Zerreissungen in der Genitalsphäre liefert der Geburtsakt. Wenn der Hymen zur Zeit der Geburt noch intakt oder nur wenig eingekerbt ist, was zuweilen bei Erstgebärenden vorkommt, so zerreisst er bei der Geburt vom freien Rand zur Basis und zerfällt in mehrere auseinanderrückende Läppchen, die nach Überhäutung der Ränder an den Lücken zu Carunculae myrtiformes werden. Die Zerreissung wird bewirkt durch den Kopf und den Thorax des Kindes, die im Vergleich zur Durebgangsöffnung zu gross sind, so dass sie den Scheideneingang enorm dehnen. Auch der Rand des Muttermundes, der beim Durchgang des kindlichen Kopfes eine gewaltige Dehnung erfährt, reisst bei allen Erstgebärenden mehrfach ein. Dies wird begreiflich, wenn man erwägt, dass der kleinste bei gebeugtem Kopf in Betracht kommende Kopfumfang der reifen Frucht $32 \mathrm{~cm}$ beträgt, während der Scheideneingang bei Primiparen zuweilen nur zwei Finger, der Muttermund von Erstgebärenden nur einen Finger durchtreten lässt.

Endlich erfolgt auch eine Abtrennung der Eihäute und des Fruchtkuchens unter Zerreissung der Decidua placentaris bei den Geburten der Raub-, Nage- und anderer Säugetiere sowie des Menschen. Beim Menschen zerreisst die vereinigte Decidua vera und reflexa schon in der Eröffnungsperiode. Bei gewissen Säugern (Raub- und Nagetieren), welche keine mit dem Chorion zusammenhängende Decidua haben, zerreisst bloss die Decidua placentaris in 
der Nachgeburtsperiode. Beim Menschen zerreisst nicht bloss die Decidua in der Ausdehnung des Chorion laeve, sondern auch der aus der Decidua serotina hervorgegangene Mutterkuchen. Bei anderen Säugern (Wiederkäuern) ziehen sich die Zotten der Lederhaut aus den Gruben der Cotyledonen heraus. Die mit der Zerreissung verbundene Blutung ist bei den Säugern gering, beim Menschen dagegen stark, weil hier die von den Uterinarterien gespeisten intervillösen Räume, die nur beim Menschen und Affen vorkommen, eröffnet werden.

Bei den physiologischen Zerreissungen handelt es sich um eine durch hochgesteigerten Innendruck bewirkte Flächendehnung, welche die Festigkeitsgrenze der betreffenden Gewebe überschreiten. Nach demselben Mechanismus geschehen auch die pathologischen Zerreissungen.

Gefässzerreissungen und Blutung.

Eine Zerreissung der oberflächlichen Kapillarien der Uterusschleimhaut mit Blutung kommt bei gewissen Säugetieren (Raubtieren und Affen) und bei Frauen wäbrend der Brunst oder Menstruation vor, bei den Wiederkäuern ist sie lokalisiert auf die Karunkeln der Uterusschleimhaut, wo letztere einen mehr papillären Bau zeigt. Bei den Hündinnen blutet es auf der ganzen Schleimhaut der Uterushörner, bei den Frauen nur aus der Schleimbaut des dreieckigen von Corpus und Fundus umfassten Cavum uteri. Diese Blutung beruht auf einer starken Füllung der betreffenden Kapillarien, deren Oberfläche durch menstruale, wenn auch oft nur partielle Zerstörung des Epithels blossgelegt ist. Sie kann sehr geringfügig, doch auch stärker, sogar erschöpfend sein und dauert meist 3-5 Tage. Bei den Säugern findet mit obigen Ausnahmen bei der Brunst keine Blutung, sondern nur eine stärkere Schleimabsonderung statt.

\section{Atrophie.}

Stehenbleiben auf einer gewissen Entwicklungsstufe, also relative Atrophie wird beobachtet bei der Thymus, der Nebenniere und dem Wolff' sehen Körper, die im Vergleich zu Nachbarorganen nur wenig im späteren Leben fortwachsen.

Eine Schrumpfung, welche zuletzt zur Obliteration fuhren kann, beobachten wir sehr häufig, besonders an gewissen Abschnitten des Gefässsystems, an manchen Strecken der Kiemenbogengefässe, dem Ductus botalli, der Nabelvene und dem Ductus venosus Arantii. 
Ausserdem tritt in dem beiden Geschlechtern zukommenden Klimakterium eine Schrumpfung der gesamten Genitalsphäre sowie der Mammae ein. Dann hört ebensowohl die Follikelreifung und -berstung wie die Menstruation auf, während beim Manne die Spermasekretion und Erektionsfähigkeit successive sich verminderen.

Eine höchste Stufe der Atrophie sehen wir unter anderem an den Ruderschwänzen der Batrachier. Diese gehören zu den ersten Organen, welche nach der Längsstreckung des Dotters aus dem Körper hervorwachsen und diesen bald bedeutend an Länge übertreffen. Wenn dann später zuerst die hinteren, dann die vorderen Extremitäten hervorgesprosst sind und sich vollständig gegliedert und zur Lokomotion genügend ausgebildet haben, bildet sich der Schwanz allmählich zurück, er schmilzt förmlich ein ; zuletzt schwindet auch der kurze Stummel an seiner Basis, und die urodele Larve wird zum anuren Batrachier. Es handelt sich in diesem Falle um eine allmähliche Verkleinerung und schliessliches Verschwinden der Gewebselemente, also um vollkommene Atrophie eines ganzen Organs.

\section{Fettmetamorphose.}

Ein interessantes physiologisches Beispiel von Atrophie mit Fettmetamorphose bieten die Corpora lutea. Nachdem diese ihre volle Entwicklung erreicht haben, beginnen sie zu schrumpfen und verwandeln sich allmählich in gelbe oder ziegelrote, bei anderen auch in blassfleischfarbene oder weissliche Gebilde (Corpora albicantia), die oft noch lange Zeit in den Ovarien deutlich erkennbar sind. Bei dieser Rückbildung treten in den Lutëinzellen, und zwar in der Umgebung der Kerne, Fettröpfehen auf, die sich vermehren und schliesslich die ganzen Zellen in einen Fettkörnchenhaufen verwandeln, wobei Kern und Protoplasma versehwinden. Auch dieser Körnchenhaufen löst sich allmählich auf.

\section{Gefässatrophie mit Thrombose.}

Eine durchaus physiologische Thrombose geschieht bei Neugeborenen in den Nabelarterien nach der Zerreissung des Nabelstranges. Dadurch entstehen zwei ungleich lange Stücke: ein längeres, das in der Nachgeburt zurückbleibt, und ein kürzeres in der Bauchhöhle des Neugeborenen. Letzteres zieht sich bei vielen Säugetieren in die Bauchhöhle zurück, entfernt sich nach innen vom Nabel und 
enthält die abgerundeten Enden der zerrissenen Arterien. Innerhalb dieses im subperitonealen Bindegewebe eingebetteten Stückes tritt nun bis zum Stamme der A. hypogastrica eine Blutgerinnung ein, und es unterliegt dann der Blutpfropf denselben Veränderungen wie ein Thrombus in anderen Arterien, d. b. es wuchern die Ausläufer der Zellen der Gefässwand in das Blutgerinnsel hinein, durchsetzen dasselbe in Form von Netzen, die eingeschlossenen Blutkörper werden gelöst und resorbiert, kurz es verschwindet die Gefässlichtung: das Gefäss wird konsolidiert.

\section{Ablösen und Absterben.}

Es gibt eine Reihe von Vorgängen, die darin übereinstimmen, dass ein Körperteil sich von den anderen und damit von dem Ganzen ablöst. Am Anfang dieser Vorgänge steht die Ablösung lebender Zellen oder Zellgruppen, welche sich allein oder nach Vereinigung mit anderen zu einem Embryo weiterentwickeln. An dem Ende der Vorgänge handelt es sich darum, dass lebende Zellen abgelöst werden, und zwar so, dass sie aufhören zu leben und aus dem Verbande des Körpers ausgeschieden werden. Dann haben wir die Nekrose vor uns.

Der einfachste Vorgang der Ablösung lebender Zellen findet sich bei den Kryptogamen und vielen niederen Tieren. Hierbei werden Sporen oder Knospen, Sprossen, d. h. einzelne Zellen oder mehrzellige Verbände, von ihren Trägern abgelöst und wachsen dann zu selbständigen Individuen aus. Dieser Art der ungeschlecht lichen Fortpflanzung steht eine andere Art gegenüber, wobei sich weibliche oder Eizellen in den Fruchtknoten oder Eierstöcken entwickeln und mit den männlichen, aus den Antheren resp. 'Testikeln abgelösten Zellen, d. h. Pollen oder Samenfäden, vereinigen, um die Anlage eines Embryo zu bilden. Aus der dureh diese Vereinigung entstandenen Stammzelle entwickelt sich dann der Embryo.

\section{Eiablösung.}

Die Ablösung des Fies von dem Mutterboden kann entweder in der Weise stattfinden, dass eine Zelle des Entoblasts sich aus dem Zellverband löst, oder dass eine Eizelle vom Ovarium sich ablöst, um entweder innerhalb des Körpers (Reptilien und Vögel) oder ausserhalb desselben befruchtet zu werden (Fische, Amphibien), oder endlich, dass der Follikel, worin das Ei enthalten ist, berstet und dasselbe austreten lässt (Säuger).

E. Pflüger, Archiv für Physiologie. Ba. 119. 
Bei den Pflanzen findet eine Ablösung des Samens mit seinen kapselartigen oder fleischigen Hüllen nach deren Reifung entweder in der Weise statt, dass sich der Samen von dem Fruchtknoten ablöst, oder dass der Stiel zerreisst, mit dem die Kapsel oder die Frucht an dem Zweige festsass, worauf dann die Samen sich aus der Kapsel lösen oder nach Zerstörung fleischiger Hüllen frei werden.

Wenn sich ganze Zellen, Zellgruppen oder ganze Gewebsstücke von dem Pflanzen- oder Tierkörper ablösen und nicht weiter wachsen, dann haben wir den

\section{Gewebstod oder die Nekrose}

vor uns. Es gehört hierher:

Das Abfallen von Pflanzenteilen. Die hier in Frage kommenden Vorgänge vollziehen sich bei Beginn einer jeden neuen Phase der Entwicklung. Wenn der Embryo mit seiner Radicula die Samenhülle gesprengt hat und nun die junge Pflanze aus ihrer Schale herauswächst, wird letztere als überflüssig gewordene Hülle zurückgelassen. Es vollzieht sich also eine Art Häutung. Wenn weiter der Keim zwischen dem einfachen oder doppelten Samenlappen hervorgesprosst ist, werden die ihrer Nährstoffe, besonders der Stärke, beraubten Kotyledonen abgeworfen, d. h. sie lösen sich von dem Stamme. Ebenso lösen sich die schützenden Deckblätter der Knospen, wenn sich die Blätter entfalten, die Deckblätter der Blüten, wenn letztere hervortreten, die Kelch- und Blumenblätter, wenn sich die Früchte entwickeln. Eine alljährlich im Herbst bei unseren einheimischen Bäumen vorkommende Erscheinung ist das Abfallen der Laubblätter, wobei die Blattstiele an einer Demarkationslinie sich von den Zweigen trennen nnd an den Ansatzstellen eine lebhafte Zellwucherung stattfindet, welche zur Bildung einer neuen Epidermis, also einer förmlichen Narbe fübrt.

Der Zahnwechsel besteht darin, dass die Milchzähne ausfallen und die an ihren Wurzeln keimenden Ersatzzähne an ihre Stelle treten. Der Vorgang spielt sich so ab, dass zuerst an einer Seite der Wurzel eine von der Wurzelspitze gegen die Krone fortschreitende Erosion entsteht. Die Einschmelzung des Zements und der Dentine wird bewirkt durch die Myeloplaxen, welche sich in einen Streifen von Granulationsgewebe entwickeln und in den angrenzenden Zahngeweben Howship'sche Lakunen aushöhlen. Die Einschmelzung rückt langsam gegen die Krone vor. Es bleiben aber 
von letzterer die basalen Partien als zackige oder zungenartige Platten erhalten. Zuletzt fällt die Krone mit ihren Wurzelanhängen aus, die Lücke wird durch Granulationsgewebe ausgefüllt, und der darunterliegende Ersatzzahn schiebt sich langsam in die Höhe.

Das Abwerfen der Geweihe. Die geweihetragenden Wiederkäuer (Rehe, Hirsche, Elentiere u. a.) werfen alljährlich im Hochsommer ihr Geweih ab, d. h. es bildet sich an der Grenze des. sogenannten Rosenstocks eine Demarkationslinie mit einer Erweichungszone des Knochengewebes, aus dem das Geweih besteht. An dieser Grenzlinie löst sich das Geweih von der Unterlage vollständig ab und es entwickelt sich dann an der Trennungsstelle Granulationsgewebe, das sich überhäutet. Es bildet sich eine neue Oberhaut mit feinen Haaren und, ausgehend von dem unterliegenden Knochen, durch subperiosteale Neubildung ein Knochenzapfen, die Anlage des neuen Geweihes: das alte Geweih fällt ab.

Mumifikation und Abfallen des Nabelstranges. Der Nabelstrang wird nach der Geburt getrennt; ein Teil bleibt am kindlichen Hautnabel bängen. Dieser Nabelschnurrest ist an dem konisehen Hautnabel gegen die Bauchhaut scharf abgegrenzt, und an dieser gehen die Kapillarien schlingenartig ineinander über. Der Peripherabschnitt, welcher keine Kapillarien enthält, wird nun bald schlaff und durch Austrocknung an den beiden ersten Tagen in einen harten Strang von dunkler Farbe verwandelt. Nun bildet sich an der Grenze von Hautnabel und Nabelstrangrest eine kreisförmige Furche, die immer tiefer einschneidet, so dass der Strangrest nur an einem Faden, d. h. an einem Gefässe hängt und schliesslich abfällt. In der Tiefe des Nabeltrichters entwickeln sich Granulationen, über welche zuletzt die Oberhaut und Lederhaut herüberwächst und das Narbengewebe des Nabels vollkommen bedeckt. Wird aber der Nabelstrang feucht gehalten, so entwickelt sich darin stinkende Fäulnis oder Gangrän.

Aus dem Mitgeteilten ergibt sich, dass die absterbenden Gewebe entweder unverändert wie Oberhautgebilde und Geweihe, oder nach ihrer Austrocknung (Mumifikation) abgestossen werden oder bei grösserem Wassergehalt und Zutritt von Fäulnisbakterien gangräneszieren.

Häutung. Wir begegnen diesem, und zwar periodischen Vorgang bereits bei den Pflanzen, indem z. B. bei den Platanen alljährlich die oberste Rindenschicht in eckige Platten zerklüftet 
und sich ablöst, worauf dann die darunterliegenden Epidermisschichten zutage treten und weiterwachsen.

Bei den Tieren beobachten wir ebenfalls Häutungen, teils beim Grösserwerden der Tiere (Insekten mit unvollkommener Metamorphose), teils beim Übergang eines Entwicklungsstadiums in ein anderes bei Tieren mit vollkommener Metamorphose. Ausserdem gibt es aber auch alljährlich wiederkehrende Häutungen, so bei Krebsen, Schlangen usw. Bei all den genannten Geschöpfen löst sich die Hornschicht der Oberhaut, deren Zellen inzwischen zu Hornzellen geworden sind, von der darunterliegenden weichen Zellschicht in Form eines zusammenhängenden Häutchens ab. Vielfach findet vorher eine Durchtränkung der unteren Sehicht statt, welche die Ablösung erleichtert.

Feder- und Haarwechsel. Bei den Vögeln seben wir in den ersten Lebenstagen ebenfalls eine Häutung der sogenannten Stoppeln, indem ein zusammenhängendes dünnes und sprödes Häutchen, welches die zusammengefaltete Federfahne scheidenartig umgibt, zum Teil mit Hilfe des Schnabels abgestreift wird. Ausserdem fällt der sogenannte Flaum, welchen die jungen Vögel mitbringen, nach kurzer Zeit aus. Endlich tritt alljährlich nach Beendigung des Brutgeschäfts eine sogenannte Mauserung ein, d. h. die weichen Zellen, welche an der Spitze des Kieles liegen und die Verbindung mit der tieferen Zellschicht der Wurzelscheide vermitteln, verhornen, der Federkiel löst sich, und die Feder fällt von selbst $a b$ oder wird durch den Schnabel des Tieres herausgerissen. Nene Federn beginnen dann alsbald hervorzusprossen.

Bei den behaarten Säugetieren erfolgt alljährlich ein sogenannter Haarwechsel, wobei die weichen Zellen der Haarzwiebel verhornen. Die nach der Härung hervorsprossenden Haare sind stärker und länger und bilden das Winterkleid.

Endlich sei hier noch erwähnt das Ansfallen der Wollhaare (Lanugo) am Stamm und den Extremitäten in den letzten 2-3 Monaten des Fötallebens bei menschlichen Früchten, das auf ähnlichen Vorgängen (Verhornung der Zellen) wie beim Haarwechsel beruht.

Als ein Beispiel von aseptischer Nekrose ist die Bildung der sogenannten Allantoisdivertikel anzuführen bei Einhufern und Dickhäutern.

Die beiden polaren Enden der Chorion-Allantoissäcke werden nämlich in frühen Entwicklungsstadien eigentümlich verändert. 
Zuerst werden die Eihäute an beschränkter Stelle trübe, dann schrumpfen sie und falten sich und stellen schliesslich dunkel gefärbte ziemlich feste Anbängsel dar, welche sich von den weiter wachsenden Eihäuten scharf abheben und von einem Kranze von Kapillarschlingen umgeben werden. Innerhalb der abgestorbenen Divertikel sind die Gefässe atrophiert, die Konturen der Zellen verwischt, die Kerne geschwunden, Fettkörnchen eingelagert und meist auch Oxalatkristalle an der Oberfläche der Membran vorhanden. Bei den Pferden ziehen sich diese Divertikel in die Allantoishöhle zurück und verwandeln sich hier in leimähnliche, gelbe Gebilde, das sogenannte Hippomanes.

Endlich sei noch erwähnt das Absterben der Ei er resp. Früchte im Innern des Mutterkörpers, besonders in den Eileitern und der Gebärmutter. Wenn mehr oder minder entwickelte $\mathrm{Frü} \mathrm{chte} \mathrm{durch}$ Kohlensäure- oder irgendeine andere Vergiftung absterben, so unterliegen sie hier, von mütterlichen Organen umsehlossen und damit vor der Einwanderung von Bakterien geschützt, gewissen Veränderungen, die man als Maceration, Mumifikation und Lithopädionbildung unterschieden hat. Solche Früchte können beliebig lange, auch bis zum Tode der Mutter im Körper zurückbleiben.

\section{Embolie.}

Die Verschleppung eines Blut- oder Eiterpfropfens, von Eiterkörpern, Krebszellen u. a. morphologisch abgegrenzten Gebilden in die Lunge oder ein entferntes Organ nennt man Embolie. Neben diesen bekannten und häufigen Formen gibt es aber eine erst neuerdings durch Veit-Halle beschriebene und von diesem als nahezu physiologisch (?) bezeichnete Form der Verschleppung organisierter Gebilde: die Zottendeportation oder Zottenembolie. Dabei werden nicht bloss Stücke von Syncytium und Zottenepithel, sondern auch grössere oder kleinere Abschnitte der Chorionzotten, die in den intervillösen Räumen frei in dem mütterlichen Blut flottieren, abgerissen. Man hat solche abgerissene und von dem Blutstrom weiterbeförderte Chorionzotten sowohl in dem Blute der Uterinvenen wie in entfernten Organen (Leber, Lungen, Nieren) wiedergefunden und von diesen die zerstreuten anämischen und nekrotischen Herde in den genannten Organen abgeleitet. Da diese Zottenstücke aseptisch sind, so veranlassen sie in ihrer Umgebung keine Eiterung, wohl aber die Bildung von sogenannten Hämolysinen und kleinen Thromben. 


\section{Höhlenwassersucht.}

Im Normalzustand kommen in den serösen Höhlen Flüssigkeitsabsonderungen vor, die das Gleiten der serösen Blätter aneinander erleichtern. Eine erhebliche Vermehrung der Flüssigkeitsmenge ist pathologisch und wird als Hydrops bezeichnet. Oben sind bereits zwei Beispiele: der Hydrocephalus und die Hydromyelie angeführt, die beide auf der Fortdauer des allerersten Embryonalstadiums der Hirnblasen und des Medullarkanals beruhen.

Ein weiteres physiologisches Beispiel ist der Graaf'sehe Follikel. Seine Höhle entwickelt sich so, dass sich zwischen den Epithelzellen des Primordialfollikels durch Auseinanderrücken derselben Interzellularräume bilden, welche zusammenfliessen urd schliesslich eine gemeinsame, den Liquor folliculi enthaltende, Höhle bilden, in welche der Keimhügel mit dem Ei hineinragt. Die Wandung der Höhle wird von der Granulosa gebildet, die einerseits eine förmliche Tapete darstellt, andererseits den Keimhügel überzieht, der das Ei enthält.

Weitere Beispiele sind die Hydrallanto is und das Hy dramnion. Erstere kommt bei denjenigen Säugern vor, deren Urachus bis zur Geburt weit offen bleibt, z. B. Wiederkäuer und Dickhäuter. Hier finden wir weite, mit Wasser gefullte Blașen, welche bis zu den Hornspitzen resp. den Eipolen reichen und dem Chorion, an einer Stelle auch dem Amnion anhaften. Diese Allantois ist sozusagen Vorblase oder Harnrezipient. Bei anderen Säugern und auch bei dem Menschen atrophiert frühzeitig die Allantois, und es bildet sich ein weiter Sack, der die Frucht nebst Nabelstrang und Fruchtwasser umsehliesst: das Amnion. Die darin enthaltene klare oder durch Wollhaare und Oberhautsehuppen getrübte und Hauttalg entbaltende Flüssigkeit ist teils fötaler Urin, teils mütterliches, durch das Amnionepithel verändertes Transsudat.

\section{Gelbsucht (Icterus).}

Eine Form desselben, den Icterus neonatorum, kann man fast schon physiologisch nennen, da ca. vier Fünftel aller neugeborenen Kinder in den ersten Lebenstagen davon befallen werden. Er dauert mehrere Tage an, zeichnet sich durch Gelbfärbung der äusseren Haut und Augenbindehaut sowie der Transsudate aus und verschwindet meist uach wenigen Tagen, ohne weitere Spuren zu hinterlassen. Nur in dem seltenen Falle einer angeborenen Atresie des Ductus choledochus tritt der Tod durch Cholämie ein. Dieser Icterus ist 
hepatogen und beruht nach meiner Auffassung darauf, dass die im Fötalleben träge abgesonderte Galle nach der Nahrungsaufnahme reichlicher abgeschieden wird und dann an der engen Gallengangmündung staut. Die an der engen Mündung stauende Galle wird nun teilweise ins Blut aufgesogen; der Rest geht ins Duodenum über und veranlasst Gelbfärbung der Stühle, während bekanntlich beim Icterus des späteren Lebens die Stühle weisslich oder grau und frei von Gallenfarbstoff sind.

\section{Konkretionbildungen.}

Die verschiedenen Sekrete werden teils als Flüssigkeiten, teils als schleimige Masse von den Drüsen ausgeschieden. Innerhalb der Drüsen oder Ausführungsgänge kann aber das Sekret erstarren, oder es können sich doch feste Körper als amorphe oder kristallinische Kugeln, eiförmige Körper und Zylinder, sogenannte Konkretionen, ausscheiden. Die Bedingungen, unter denen dies geschieht, sind im Grunde dieselben wie die von Niederschlägen in Flüssigkeiten: 1. Eindickung, 2. Abkühlung und 3. Neubildung chemischer, im Wasser unlöslicher Verbindungen aus den in dem Lösungsmittel enthaltenen Substanzen. Die Konkretionen im Tierkörper kommen, vorzugsweise auf dem zweiten und dritten Wege zustande. Zn letzteren gehören die Speichel-, Darm-, Gallen- und Harnsteine. Für diese Konkretionsbildungen, die allgemein als pathologisch betrachtet werden, gibt es nur ein physiologisches Paradigma: nämlich die ganz normale Bildung griesartiger Harnsäureausscheidung bei Reptilien und Vögeln innerhalb der Harnwege. Diese pulverförmigen Massen werden dann durch die Harnleiter in die Kloake geführt und mit dem Kot ausgeschieden.

Der Harnsäureinfarkt, der in den geraden Harnkanälen der Nieren bei Sektionen von neugeborenen Kindern der ersten Lebenswochen sich mindestens sehr häufig vorfindet und im Urin als pulverförmiger Bodensatz nachgewiesen werden kann, ist als nahezu physiologischer Vorgang ebenfalls hierher zu rechnen. Er führt zuweilen $\mathrm{zu}$ den Nierensteinen der Kinder.

\section{Verkalkung.}

Ablagerung von Kalkkarbonat und -phosphat auf die Oberfläche und in die Wand von Organen, teils in Form von Körnern und teils als Platten wird pathologischerweise vielfach beobachtet. Wohl 
am häufigsten ist die pathologische Verkalkung der Media der Arterien, der Herzklappen und Herzwände, ferner der Fasergeschwülste. Im Pflanzen- und Tierreich kommen durchaus physiologische Kalkablagerungen massenhaft vor. Wir erwähnen nur die Kalkablagerungen in den Flechten, in den Korallen wo sie vielverzweigte, baumartige Stöcke darstellen, in den Schalen der Muscheln, in den Gehäusen der Schnecken, wo sich Kalksalze in dem von der Hautoberfläche abgesonderten Schleim niederschlagen und die alljährlich gebildeten Massen durch Jahresringe von den früher entstandenen abgrenzen. (Ähnlich wie Kalksalze werden auch Silikate auf der Oberfläche oder im Innern der Organe abgelagert bei den Kieselalgen, den Riedgräsern, den Kieselschwämmen usw.)

\section{Verknöcherung.}

Für die Verknöcherungen in den Muskeln und Sehnen, welche als Myositis ossificans bekannt ist, haben wir ein physiologisches Vorbild in den Verknöcherungen der Sehnen von Extremitätenmuskeln vieler Vögel, zumal Hühnervögel. Diese werden schon frühzeitig in starre Platten oder Stäbe verwandelt und zeigen vollkommene Knochenstruktur.

\section{Erbrechen.}

Wenn man unter Erbrechen das Zurücktreten der verschluckten und in die Speiseröhre oder in den Magen gelangten Nahrung bis zur Mundhöhle und nach aussen versteht, so kommen mehrere physiologische Vorbilder in betracht:

das Herauswärgen der in den Kropf vorgedrungenen und durch dessen Sekrete erweichten Pflanzensamen zur Fütterung der Jungen, wie wir dies z. B. bei den Tauben beobachten,

das Auswerfen von Gewöllballen, d. h. von Haaren und Knochen der gefressenen Tiere nach Auflösung des Fleisches im Kropfe, wird bei den Raben und Raubvögeln beobachtet,

das Erbrechen von Knochen, die im Magensaft unlöslich sind, geschieht ca. alle 4 Wochen bei den Hyänen, jedenfalls bei den in den zoologischen Gärten gehaltenen Tieren,

beim Widerkäuen der danach benannten Säugetierklasse wird das zerkaute Futter, nachdem es in den Pansen und die Haube übergetreten und hier unvollkommen verdaut ist, durch die Speiseröhre in die Mundhöhle zurückgedrängt, nochmals gekaut und wieder 
versehluckt, um zuletzt im Psalter und Labmagen möglichst vollständig verdaut $\mathrm{zu}$ werden.

Es ist bekannt, dass die Arbeiterbienen mittelst ihrer Rüssel den in den Nektarien der Blüten enthaltenen Honigsaft aussaugen und nach der Rückkehr in den Bienenstock in die aus Wachs hergestellten Zellen der Waben als Honig entleeren. Honig ist also der erbrochene Mageninhalt der Bienen.

Das pathologische Erbrechen kommt bei dem menschlichen Weibe in $40 \%$ der Fälle in einer oder der anderen Schwangerschaft vor. Auch bei manchen domestizierten Tieren, so bei Hündinnen und Katzen, tritt öfters Erbrechen ein während der Gravidität. Früher hat man den Vomitus gravidarum als einen von den sensiblen Genitalnerven eingeleiteten Reflex betrachtet; neuerdings neigt man zur Ansicht, dass in der Gravidität gewisse noch unbekannte Produkte des fötalen Stoffwechsels das Brechzentrum erregen.

Wenn wir die Ergebnisse der mitgeteilten Beobachtungen zusammenfassen und durch allbekannte Beispiele ergänzen, so kommen wir zu folgenden Schlusssätzen:

1. Die bei allen Individuen einer Art vorkommenden anatomischen Befunde und physiologischen Vorgänge (Verdauung, Atmung, Lymph- und Blutbildung, Blutumtrieb und Drüsenausscheidung sowie Ortsbewegung u. a.) sind als normale oder physiologische zu bezeichnen.

2. Gewisse Prozesse, die allgemein als pathologiseh gelten, sind nicht ausschliesslich als solche anzuerkennen, sondern kommęn regelmässig bei einem oder bei beiden Geschlechtern in gewissen Zeiten des embryonalen oder freien Lebens vor. Hierher gebören die in dieser Arbeit speziell abgehandelten Vorgänge: Zerreissung, Blutung, Hypertrophie, Atrophie, Nekrose, Steinbildung usw. Man kann sie füglich physiologisch-pathologische Vorgänge nennen.

3. Eine dritte Gruppe enthält alle bei den einzelnen Individuen einer Art nur ausnahmsweise vorkommenden Vorgänge, für welche bis jetzt noch keine physiologischen Vorbilder gefunden sind. Dies sind die pathologischen Prozesse im engeren Sinne.

Ich habe in dieser Arbeit die zweite Gruppe eingehend abgehandelt, und zwar aus zwei Gründen: 
624 F. A. Kehrer: Die Grenzen der Physiologie und Pathologie.

1. wollte ich zeigen, dass eine scharfe Grenzbestimmung von Physiologie und Pathologie unmöglich ist, denn derselbe bei einem Geschlecht oder in gewissen Lebensaltern durchaus physiologische Vorgang wird in einem anderen Falle als entschieden pathologisch zu betrachten sein;

2. machte ich auf dieses Grenzgebiet aufmerksam, weil das Studium der hier aufgezählten Erscheinungen geeignet scheint, uns Aufklärung zu geben über Entstehung und Verlauf der pathologischen prozesse im engeren Sinne. 